\title{
Percepción de la imagen corporal y prácticas alimentarias entre indígenas Mayas de Yucatán, México
}

\author{
Body image perception and food \\ practices of Mayan indigenous \\ of Yucatan, Mexico
}

\begin{abstract}
The objective of this study was to assess body image perception and feeding practices of indigenous Mayan of two different rural areas of Yucatan. Eight men and 2 women from 10 families of the community of Xanlah (corn forming area) and 8 women and 3 men from another community called Mucuyche (Henequen plantation) were selected; height and weight were measured to determine body mass index (BMI). Usual dietary practices and in-depth interviews were done to know their self-image perception. The answers showed similarities in both communities, being "chubby" is part of their cultural context and considered "normal" and food selection was not based on nutritional value, but on the status, recognition and prestige which these foods represent. It's essential that the nutrition policies are implemented with strategies that are pertinent to the social and cultural context of the country.

Key words: Perception; traditional; feeding; obesity; healthy.
\end{abstract}

\section{INTRODUCCIÓN}

La obesidad es actualmente uno de los principales problemas de salud pública que enfrenta la población; fenómeno que incluye a los países industrializados como a los en desarrollo (1).Tiene un gran impacto en la salud y es un factor de riesgo para el desarrollo de enfermedades crónicas degenerativas no transmisibles (2) como hipertensión arterial, diabetes mellitus tipo 2, riesgo cardiovascular, dislipidemias, entre otras (3).

La Organización Mundial de la Salud (OMS) afirmó que 1,200 millones de personas en todo el mundo tienen problemas de sobrepeso y obesidad; lo que es equiparable al número de personas que sufren desnutrición. Los estudios epidemiológicos muestran que $55 \%$ de la población adulta presenta sobrepeso y $22 \%$ obesidad (4). El Fondo de las Naciones Unidas para la Infancia (UNICEF) afirma que la obesidad constituye un problema severo en México debido a que ocupa el segundo lugar a nivel mundial en la población adulta; y especifica que uno de cada tres mexicanos la padecen (5).

La alimentación, es un proceso sensorial y cognitivo que comprende procesos y mecanismos para elaborar y generar juicios sobre los otros y sobre sí mismo. Es el proceso de organizar e interpretar información sensorial para darle significado. Las personas son centro de acción e intención (6), por tanto,
Odette Pérez I. (1)

Damaris Estrella (2)

(1) Licenciatura en Nutrición, Facultad de Medicina, Universidad Autónoma de Yucatán, México. (2) Licenciatura en Rehabilitación, Facultad de Medicina, Universidad Autónoma de Yucatán, México.

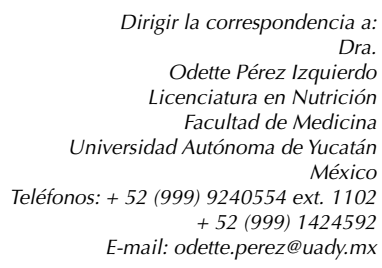

Este trabajo fue recibido el 19 de Marzo de 2014 y aceptado para ser publicado el 12 de septiembre de 2014 .

estudiar la percepción de las personas sobre lo que comen y cómo se alimentan no es una tarea fácil, ya que según Mauss es un hecho social total, entendiendo que todas las áreas de la cultura y tipos de instituciones encuentran en él expresión simultánea y lo influyen de algún modo (7). Por lo que analizar el hecho alimentario o el acto de comer, revela tanto la naturaleza como la estructura de un orden social.

Por ello, uno de los factores condicionantes del peso corporal que es necesario tomar en cuenta es la percepción que la propia persona tiene de su imagen corporal (8). El cuerpo, se construye en parte por la percepción que los seres humanos tienen de él; es una construcción simbólica, no una realidad en sí mismo y de ahí que la mirada que busca darle sentido no sea igual entre las diferentes sociedades (9). Es decir, la percepción de la imagen corporal que las personas tienen de ella, va a depender directamente de los atributos que la sociedad y la cultura le otorguen (10).

En Latinoamérica y México son escasas las investigaciones realizadas sobre percepción de la imagen corporal en población rural e indígena. Entre las pocas aproximaciones, en Chile, Atalah et al (11), compara la percepción que las personas tienen sobre su estado de nutrición con las Normas del Ministerio de Salud. Los resultados encontrados demuestran 
que prácticamente la mitad de la población analizada tenía una percepción errada de su estado nutricional, esto es, una sobrestimación del peso (60\%) y un grupo más pequeño, una subestimación, destacando que sólo un tercio de las personas obesas se consideró como tal. Esto puede deberse a que la percepción cultura y simbólica de lo que representa el cuerpo, así como también a la percepción imaginaria y de la salud (12).

En el caso de México, Pérez-Gil y Romero (9) realizaron un estudio con mujeres de siete comunidades del estado de Oaxaca: tres comunidades pertenecientes a la Sierra Juárez (Yacochi, Laxopa y Yahuio) y cuatro comunidades de la Costa Oaxaqueña (Chacalapa, La Luz, Palma Sola y Santa Rosa), en donde la autopercepción del cuerpo de las mujeres (modelo percibido), al mostrar los modelos anatómicos, $41.1 \%$ de las encuestadas en la sierra percibieron que tenían algún grado de desnutrición, mientras que para el caso de las mujeres de la costa, sólo $26.4 \%$ se auto-percibieron delgadas o desnutridas.

Se observó que un porcentaje significativo de las mujeres eligieron una figura delgada, tal vez pudiera ser por la presión social hacia tener una silueta esbelta.

Las investigaciones en México que aborden el estudio de la percepción de la imagen corporal en población indígena y sus prácticas alimentarias realmente son escasas, por ello, la importancia de llevar a cabo esta investigación en dos poblados mayas de Yucatán pertenecientes cada uno a dos regiones productivas del estado, permitirá identificar el concepto de la imagen corporal desde su cultura, relaciones familiares y sociales dentro de cada comunidad, así como practicas alimentarias que tienen en la actualidad de acuerdo a los sistemas productivos de cada una de las regiones, los cambios y adaptaciones que han tenido estos.

El objetivo de este estudio fue conocer la percepción de la imagen corporal y las prácticas alimentarias de indígenas mayas de dos zonas rurales de Yucatán, México.

\section{SUJETOS Y MÉTODOS}

Descripción de las comunidades de estudio

El estudio se llevó a cabo en dos comunidades indígenas mayas del estado de Yucatán. La primera localidad Xanláh, pertenece al municipio de Chankom; se encuentra localizada en la región milpera, ubicada en el cono sur-oriente del estado. Tiene una población de 406 habitantes de los cuales $85 \%$ habla lengua maya (12) y posee un índice de marginación muy alto (13).

En esta zona el hombre se concibe milpero, como tal reconoce que el monte le pertenece a seres míticos, a quienes tiene que pedir permiso para arrancarle el fruto de la tierra (14). La producción de la milpa es además un ritual, la cual puede considerarse tradicional, en la medida en que la forma social de relación y apropiación de la naturaleza está centrada en ella, y por tanto, se asocia con actividades productivas y culturales.

La segunda comunidad, llamada Mucuyché, situada en la región henequenera, zona norponiente del estado y pertenece al municipio de Abalá. Tiene una población aproximada de 494 habitantes y $87.2 \%$ habla lengua maya (12). La actividad productiva principal es la agricultura junto con el sector secundario que poco a poco la va desplazando. Conserva muchas de sus tradiciones y su índice de marginación es alto (13).
Esta región productiva fue transformada drásticamente en las últimas décadas; durante años anteriores tuvo un gran auge la producción de henequén conocido como "el oro verde" (el cual en la actualidad prácticamente se encuentra casi extinto). Actualmente, los nuevos modelos de orientación social, nuevas instituciones económicas y socio-políticas enmarcados en el mundo industrial y comercial, provocaron cambios: en la dinámica de la economía campesina, en los patrones productivos, en la organización del trabajo familiar y en la concepción cultural de la producción agrícola (15).

\section{MÉTODO}

El estudio es de tipo cuali-cuantitativo, descriptivo, en el cual participaron 51 familias, y al aplicar los criterios de inclusión: hombres y mujeres adultos, casados (as), de 18 años o más edad, se seleccionaron 21 personas de ambas comunidades para el desarrollo del estudio. Sólo se excluyeron a quienes poseían alguna discapacidad mental o que no aceptaran participar en el estudio. Se consideró para el estudio la edad, el sexo, nivel socioeconómico, escolaridad y la ruralidad, así como la lengua, es decir, si las personas hablaban maya, español o ambos. La fórmula que se empleó para el tamaño muestral fue aleatorio simple:

$\mathrm{n}=\mathrm{n}_{\mathrm{o}} /(1+\mathrm{no} / \mathrm{N})$

Donde $n_{0}=\left(p^{*} q^{*} z 2\right) / d 2$.

Se estimó una tasa de no respuesta del $10.0 \%$ para un tamaño mínimo estimado de muestra de 51 personas, con el fin de aplicar las mediciones antropométricas.

Para ello se realizaron mediciones de peso $(P)$ con una báscula de piso portátil marca SECA modelo 750 con capacidad de $150 \mathrm{~kg}$; la talla (T), se determinó con un estadímetro modelo 213 marca SECA. Las medidas de peso y talla se efectuaron a las 51 familias para identificar el Índice de Masa Corporal (IMC) (Kg/m2). La toma de medidas antropométricas fue realizada por la responsable del estudio, se llevaron a cabo en la casa del responsable de salud que coordina el Programa Oportunidades ${ }^{1}$-en Mucuyché- $y$, en la localidad de Xanláh la toma antropométrica se realizó en la Unidad Médica Rural del pueblo. Una vez obtenidas las medidas antropométricas se procedió al cálculo y para la clasificación del IMC, se tomaron como referencia los puntos de corte establecidos por la OMS (16): desnutrición de primer grado de 17.0 a 18.4; desnutrición segundo grado de 16 a 16.9 y de tercer grado, menor a 16; peso normal, de 18.5 a 24.9, sobrepeso de 25 a 29.9, obesidad grado 1, de 30 a 34.9 , grado 2 , de 35 a 39.9 y obesidad grado 3 , mayor de $40(17,18)$; los resultados del IMC se presentan en porcentajes, para el análisis de los resultados cualitativos se realizaron descriptivamente.

Posteriormente se seleccionaron a 10 familias (8 mujeres y 2 hombres) de la comunidad de Xanláh, y a 11 familias (8 mujeres y 3 hombres) de la localidad de Mucuyché; para conocer la percepción de su imagen corporal, aplicar las entrevistas en profundidad y dieta habitual.

La percepción de la imagen corporal se evaluó a través de la selección de la figura 1, que según su percepción y gusto, correspondía a su imagen corporal actual. Las figuras se eligieron de entre nueve diferentes siluetas de mujeres y hombres, en diferentes rangos y finalmente, se les preguntó la razón por la cual seleccionaron esa figura (esta pregunta forma parte de la entrevista en profundidad).

1 Es un programa integral destinado a la población de extrema pobreza, que padecen los más altos índices de desnutrición, enfermedades básicas curables y deserción escolar. Tiene como objetivo apoyar a las familias que viven en condiciones de pobreza extrema. El fin es el incrementar las capacidades de sus integrantes y ampliar sus alternativas para alcanzar mejores niveles de bienestar, a través del mejoramiento opciones en educación, salud y alimentación, además de contribuir a la vinculación con nuevos servicios y programas de desarrollo que propicien el mejoramiento de sus condiciones socioeconómicas y de calidad de vida. 
La entrevista en profundidad (que se aplicó de manera oral) estuvo integrada por las siguientes preguntas: 1) ¿cómo considera su cuerpo?, 2) ¿qué piensa del sobrepeso y obesidad? y 3) ¿qué alimentos cree que provocan el sobrepeso y obesidad? A las cuales se les realizó un análisis de contenido.

Las personas seleccionadas se dividieron en grupos de edad de 18 a 29 años, de 30 a 59; y de 60 y más, se dividió por grupo de edad para observar la percepción que cada uno de ellos tiene respecto al valor que le otorgan a la alimentación y desde luego a los alimentos que consumen.

Se les pidió su consentimiento verbal para visitarlos(as) en su domicilio; se les explicó con detalle en qué consistía el estudio y el objetivo de realizar cada una de las actividades. Todo esto se realizó en los días y horas que ellos(as) establecieron.

Esta investigación fue aprobada por el Comité de Docencia del Postgrado de El Colegio de la Frontera Sur (ECOSUR), clasificando este estudio que no pone en riesgo la salud de las personas participantes en él.

\section{RESULTADOS}

Composición corporal de Mucuyché

Se encontró que $23 \%$ de las personas presenta un peso normal, $41 \%$ sobrepeso, $23 \%$ obesidad grado I y sólo $6 \%$ obesidad grado II; $7 \%$ de los participantes no se lograron contactar porque al asistir a su domicilio para las mediciones no se encontraban.

Al sumar de sobrepeso y obesidad, $70 \%$ de los (as) participantes presentaban alguno de estos dos estados, representando un factor de riesgo para la aparición de enfermedades crónico degenerativas. Al segregar los datos por género se encontró que $35 \%$ de las mujeres presentaban sobrepeso y obesidad, al igual de los varones.

\section{Composición corporal Xanláh}

En esta localidad se encontró que $2 \%$ presentaba bajo peso, $37 \%$ peso normal, $37 \%$ sobrepeso, $5 \%$ obesidad grado I y $11 \%$ obesidad grado II; no se obtuvieron los datos del $8 \%$ de

Siluetas de la imagen corporal que se les mostró a hombres y mujeres encuestados(as) para que seleccionaran una.
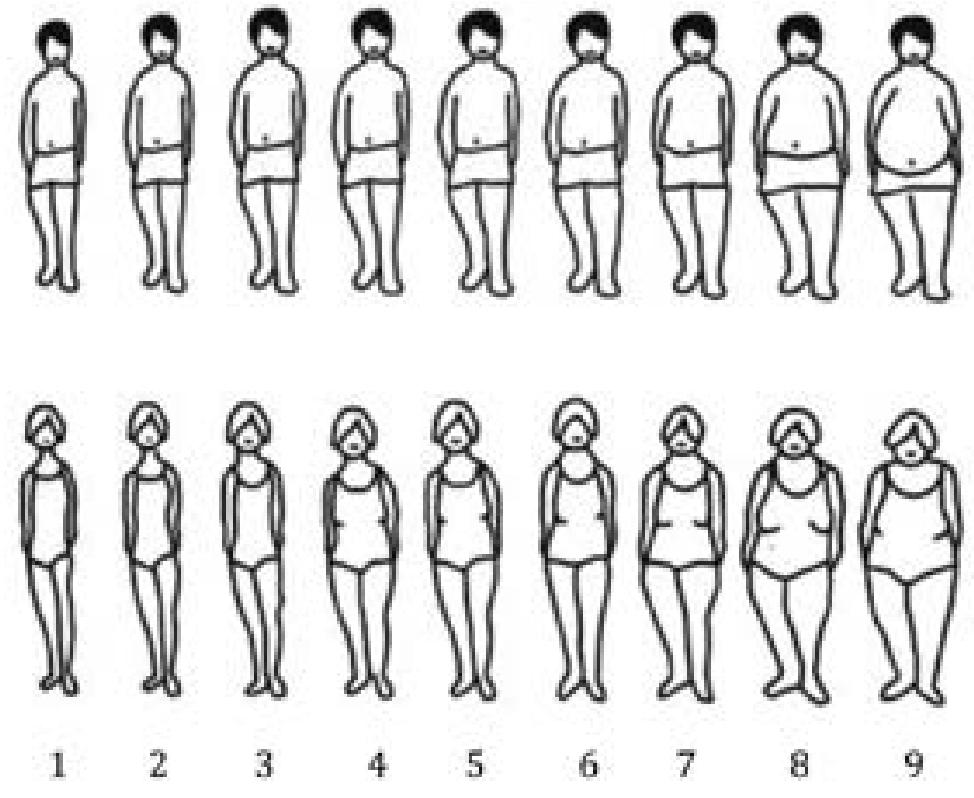

8

9

Sorensen TIA, Stunkard AJ,Teasdale TW, Higgins MW. The accuracy of report of weight:

Children recall of their parent's weight 15 years earlier. Int J Obes 1983; 7:1 15-122

Los modelos se clasificaron ajustándolos de acuerdo a los criterios de IMC y se categorizaron de la siguiente manera:

$\begin{array}{cc}\text { Modelo } 1 & \text { Desnutrición 3 } \\ \text { Modelo } 2 & \text { Desnutrición 2 } \\ \text { Modelo 3 } & \text { Desnutrición 1 } \\ \text { Modelo 4 } & \text { Normal } \\ \text { Modelo 5 } & \text { Sobrepeso } \\ \text { Modelo 6 } & \text { Obesidad 1 } \\ \text { Modelo 7 } & \text { Obesidad 2 } \\ \text { Modelo 8 y } 9 & \text { Obesidad 3 }\end{array}$




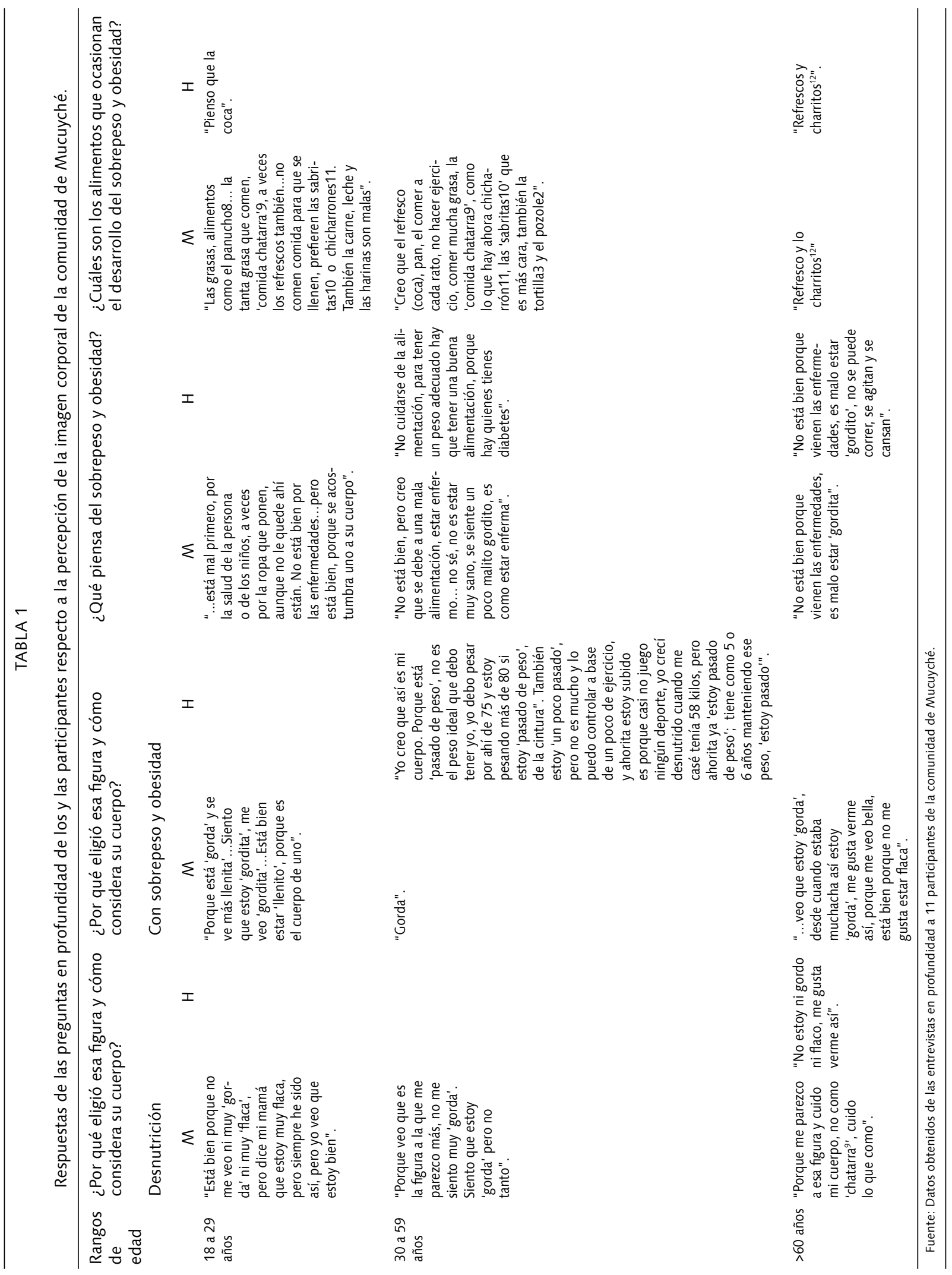


la población, ya que al asistir a su domicilio no se encontraban.

El 53\% presenta sobrepeso y obesidad, registrándose una diferencia significativa entre ambas comunidades. En cuanto a las mujeres se encontró que $32 \%$ presenta sobrepeso y obesidad, en cuanto a los hombres sólo representa el $21 \%$.

Percepción de la imagen corporal Mucuyché En esta comunidad se entrevistaron a 11 personas (hom- bres y mujeres). Del grupo de 18 a 29 años (3 mujeres); 2 de ellas seleccionaron la imagen que representa a la figura con sobrepeso y una, eligió la imagen normal (tabla 1).

Percepción de la imagen corporal Xanláh

El primer grupo (mujeres de 18 a 29 años) está conformado por 4 mujeres, de las cuales 3 perciben su imagen corporal con desnutrición y sólo una se percibe obesa. (tabla 2).

\section{TABLA 2}

Respuestas de las preguntas en profundidad de los/las participantes respecto a la percepción de a imagen corporal de la comunidad de Xanláh.

\begin{tabular}{|c|c|c|c|c|c|c|c|}
\hline \multirow[t]{2}{*}{$\begin{array}{l}\text { Rangos } \\
\text { de } \\
\text { edad }\end{array}$} & \multicolumn{2}{|c|}{$\begin{array}{l}\text { ¿Por qué eligió esa figura y } \\
\text { cómo considera su cuerpo? }\end{array}$} & $\begin{array}{l}\text { ¿Por qué eligió esa figura y } \\
\text { cómo considera su cuerpo? } \\
\text { Con sobrepeso y obesidad }\end{array}$ & \multicolumn{2}{|c|}{$\begin{array}{l}\text { ¿Qué piensa del sobrepeso y } \\
\text { obesidad? }\end{array}$} & \multicolumn{2}{|c|}{$\begin{array}{l}\text { ¿Cuáles son los alimentos } \\
\text { que ocasionan el desa- } \\
\text { rrollo del sobrepeso y la } \\
\text { obesidad? }\end{array}$} \\
\hline & $M$ & $\mathrm{H}$ & $M$ & $M$ & $\mathrm{H}$ & $M$ & $\mathrm{H}$ \\
\hline $\begin{array}{l}18 \text { a } 29 \\
\text { años }\end{array}$ & $\begin{array}{l}\text { Porque no está } \\
\text { tan 'llena ni tan } \\
\text { gordita'... Porque } \\
\text { está media } \\
\text { flaca, y así creo } \\
\text { que tengo mi } \\
\text { cuerpo... Porque } \\
\text { así 'estaba' mi } \\
\text { cuerpo, todavía } \\
\text { veo mi cuerpo } \\
\text { así, está un poco } \\
\text { 'gordita'". }\end{array}$ & & $\begin{array}{l}\text { "mmmmmm... } \\
\text { porque estoy 'gor- } \\
\text { da', más o menos } \\
\text { 'gordita', me veo } \\
\text { 'gorda'...". }\end{array}$ & $\begin{array}{l}\text { "Bueno a nosotros } \\
\text { nos explican cuándo } \\
\text { vamos en la clínica } \\
\text { que hay que tener } \\
\text { una buena alimen- } \\
\text { tación, balanceada } \\
\text { y además nos dicen } \\
\text { que lo flaco no es } \\
\text { bueno y tampoco lo } \\
\text { gordo. } \\
\text { Yo creo que está bien } \\
\text { estar gordito, porque } \\
\text { así no te puede } \\
\text { dar enfermedad... } \\
\text { Es un riesgo de } \\
\text { enfermedad, porque } \\
\text { el sobrepeso puede } \\
\text { dar diabetes, es malo } \\
\text { estar gordito". }\end{array}$ & & $\begin{array}{l}\text { "Pues... si comen } \\
\text { diario carne, hay } \\
\text { algunos que toman } \\
\text { vitaminas y hace } \\
\text { que engorde uno... } \\
\text { También el huevo, } \\
\text { las grasas de la car- } \\
\text { ne, las tortillas3... } \\
\text { Pero me dijeron } \\
\text { que las verduras } \\
\text { como la chaya4 y } \\
\text { la calabaza, que el } \\
\text { refresco...El aceite, } \\
\text { la 'tortiha', el pan, } \\
\text { las barras de pan } \\
\text { francés". }\end{array}$ & \\
\hline $\begin{array}{l}30 \text { a } 59 \\
\text { años }\end{array}$ & $\begin{array}{l}\text { "Porque no está } \\
\text { alimentada bien } \\
\text { jejeje. La que 'al- } \\
\text { canza' es la que } \\
\text { come, creo que } \\
\text { no me alimento } \\
\text { bien, pues no } \\
\text { tenemos dinero y } \\
\text { hay que comprar } \\
\text { maíz y frijol, el } \\
\text { frijol cuesta } 24 \\
\text { pesos..." }\end{array}$ & $\begin{array}{l}\text { "Porque no } \\
\text { está gordo" }\end{array}$ & $\begin{array}{l}\text { "Pos' creo que así es } \\
\text { mi cuerpo, porque } \\
\text { estoy 'gorda' } \\
\text { "Pues bueno yo veo } \\
\text { que no es normal, } \\
\text { porque engordarse } \\
\text { es muy malo, yo } \\
\text { veo que no estoy } \\
\text { 'gordo'". }\end{array}$ & $\begin{array}{l}\text { "Pues 'dicen' que no } \\
\text { es bueno, y pues creo } \\
\text { no, 'pero estoy gor- } \\
\text { da' jejeje... Pues los } \\
\text { 'gorditos' 'pus' dicen } \\
\text { que por obesidad, di- } \\
\text { cen que porque están } \\
\text { alimentados ellos se } \\
\text { ven bonitos porque } \\
\text { están gorditos, a mí } \\
\text { no me gusta porque } \\
\text { algunos salen al sol y } \\
\text { se agitan..." }\end{array}$ & $\begin{array}{l}\text { "Que la persona } \\
\text { no sea 'muy gor- } \\
\text { do', pero que no } \\
\text { se pase, porque } \\
\text { 'dicen' de la } \\
\text { obesidad, pues } \\
\text { ahí ya no... gor- } \\
\text { do no es malo, } \\
\text { a mí me gusta } \\
\text { verme así para } \\
\text { mi es normal, } \\
\text { con el trabajo } \\
\text { que hacemos } \\
\text { no podemos } \\
\text { engordar". }\end{array}$ & $\begin{array}{l}\text { "Pos creo que la } \\
\text { 'tortiha' como mu- } \\
\text { cho quedé 'gorda', } \\
\text { dice el doctor que } \\
\text { baje de peso, creo } \\
\text { que es normal el } \\
\text { cuerpo de uno no } \\
\text { estar muy gordo, } \\
\text { no estar muy flaca, } \\
\text { no sé, creo que } \\
\text { tomo mucho coca } \\
\text { queda uno 'gorda'". }\end{array}$ & \\
\hline$>60$ años & $\begin{array}{l}\text { "Como regular... } \\
\text { creo (persona que } \\
\text { percibe su cuerpo } \\
\text { con desnutri- } \\
\text { ción)..." }\end{array}$ & & $\begin{array}{l}\text { "... Porque está "más } \\
\text { gorda, más salud, } \\
\text { tiene más comida"... } \\
\text { Porque de antes } \\
\text { estamos macizos } \\
\text { antes tenía bonito mi } \\
\text { cuerpo, pero ahorita } \\
\text { nada...". } \\
\text { "Yo veo mi cuerpo } \\
\text { bello, yo veo mi } \\
\text { cuerpo regular ni muy } \\
\text { gorda ni muy flaca, } \\
\text { 'gorda' está feo... }\end{array}$ & "Se ve el cuerpo feo" & $\begin{array}{l}\text { "Es bueno estar } \\
\text { 'gordito', por- } \\
\text { que tu cuerpo } \\
\text { esta fuerte, } \\
\text { cuando te } \\
\text { enfermas quedas } \\
\text { flaco..." }\end{array}$ & & \\
\hline
\end{tabular}

Fuente: Datos obtenidos de las entrevistas en profundidad a 10 participantes de la comunidad de Xanláh. 
Consumo habitual de alimentos

La dieta habitual para identificar las preparaciones que forman parte del consumo diario de la población, así como el tipo de alimentos que la integran y los horarios (19).

La dieta habitual de las familias estudiadas de las dos comunidades estaba integrada por desayuno, almuerzo (media mañana), comida y cena; y una que otra colación por la mañana o por la tarde.

En la comunidad de Xanláh, lo que frecuentemente desayunan es café con pan francés, pan dulce o galletas; a media mañana las personas consumen la bebida tradicional conocida como pozole ${ }^{2}$; la comida la integra el frijol, las tortillas ${ }^{3}$ hechas a mano y el huevo con algunas verduras (principalmente tomate, cebolla, chile y chaya ${ }^{4}$ ), así como agua de sabor (se usa polvo para preparar el agua). La cena la conforma lo que sobra de la comida, o bien toman café con pan francés, pan dulce o galletas; muy pocas familias consumen huevo con frijoles y tortilla ${ }^{3}$.

La comunidad de Mucuyché consume en el desayuno café solo o acompañado con leche, huevo con frijol y tortilla. A media mañana se consume el pozole ${ }^{2}$, lo mismo que en la comunidad Xanláh, debido a que es una bebida tradicional relacionada con la fuerza y energía para quien la toma. La comida está compuesta por frijol, tortilla, el guisado que puede ser pollo o cerdo, algunas personas consumen huevo, tortillas ${ }^{3}$ (algunas hechas a mano y otras de máquina) refresco embotellado, agua de sabor o bebida preparada con alguna fruta que tenga en su solar ${ }^{5}$ (esto es mínimo), así como chile que acompaña la comida. En cuanto a la cena, la mayor parte de las familias consumen lo que sobra de la comida, pero puede haber una mayor diversidad de preparaciones, desde burritas $^{6}$ hasta tortas 7 huevos, entre otros.

Las preparaciones que forma parte de la dieta habitual se presentan en la tabla 3, observándose que la dieta en las dos comunidades es muy similar, aunque la cena y las colaciones vespertinas son más variadas en la comunidad de Mucuyché, tal vez por ello la mayor cantidad de sobrepeso y obesidad.

\section{DISCUSIÓN}

El estudio presenta los resultados encontrados en tres dimensiones, desde el IMC, la percepción de la imagen corporal y su relación con la dieta habitual, por comunidad. Como se contextualizó desde inicio las comunidades son diferentes desde un análisis agrícola, pero comparten todas las similitudes tradicionales y culturales.

En la dimensión del IMC se encontró que el sobrepeso y la obesidad son mayores en la comunidad de Mucuyché en comparación con Xanláh, lo mismo sucedió por género, es mayor la obesidad en mujeres que en hombres, en las dos localidades, pero sin perder de vista que es más acentuada en la primera localidad. Estos resultados son similares a los encontrados en la Encuesta Nacional de Salud y Nutrición (ENSANUT), 2006, en donde se reporta que el porcentaje de obesidad es mayor en la mujeres (68\%) que en hombres (59\%) (20). Esto tal vez se debe a que la alimentación que tienen es menos industrializada, tal y como lo revela el estudio realizado

\section{TABLA 3}

Alimentos y preparaciones que conforman la dieta habitual de las dos comunidades.

\begin{tabular}{|c|c|c|c|c|}
\hline Desayuno & Colación matutina & Comida & Colación vespertina & Cena \\
\hline \multicolumn{5}{|l|}{ Comunidad Xanaláh } \\
\hline $\begin{array}{l}\text { Huevo con frijol y tortillas } \\
\text { hechas a mano. } \\
\text { Café con pan francés o } \\
\text { galletas. }\end{array}$ & Pozole $^{2}$ & $\begin{array}{l}\text { Huevo con frijol y tortillas, } \\
\text { acompañada de refresco de } \\
\text { sabor o refresco embotellado. }\end{array}$ & $\begin{array}{l}\text { Muy pocas personas con- } \\
\text { sumen frutas de temporada } \\
\text { que tienen en sus patios o } \\
\text { pan. }\end{array}$ & $\begin{array}{l}\text { Lo que sobra de la comida, } \\
\text { es decir, se vuelve a cenar } \\
\text { huevo con frijol y tortillas6, } \\
\text { acompañado de café con pan } \\
\text { francés, galletas o pan dulce. }\end{array}$ \\
\hline \multicolumn{5}{|l|}{ Comunidad Mucuyché } \\
\hline $\begin{array}{l}\text { Huevo con tortillas }{ }^{6} \text { hechas a } \\
\text { mano o de máquina. } \\
\text { Café con pan francés, pan } \\
\text { dulce o galletas }\end{array}$ & Pozole $^{7}$ & $\begin{array}{l}\text { Frijol con arroz acompañado } \\
\text { de guisado con carne de pollo } \\
\text { o carne de cerdo, con algunas } \\
\text { verduras, tortillas }{ }^{6} \text { y chile } \\
\text { habanero' } \\
\text { Refresco de sabores o refresco } \\
\text { embotellado. } \\
\text { También las pastas forman } \\
\text { parte de la dieta habitual de la } \\
\text { población. }\end{array}$ & $\begin{array}{l}\text { Algunas personas consumen } \\
\text { papas industrializadas, pan, } \\
\text { atole }^{2} \text { y pozole. }\end{array}$ & $\begin{array}{l}\text { La cena de esta comuni- } \\
\text { dad es muy variada, está } \\
\text { conformada por los alimentos } \\
\text { que sobran de la comida, así } \\
\text { como tortas, sándwiches, } \\
\text { panuchos }^{2} \text {, huevo, café y pan. }\end{array}$ \\
\hline
\end{tabular}

Fuente: Dieta habitual realizadas a las 21 personas entrevistados, resultados del trabajo de campo.

2 Es una bebida de la gastronomía maya basado en maíz y consiste en el cocimiento del nixtamal una noche, se lava y se muele en el molino a mano. Se agrega sal o azúcar, según el gusto. Es la comida del campesino en el monte.

3 Alimento de forma circular y plano que se hace con masa de harina de maíz o trigo prensada, o hecha a mano, que se cocina al fuego y se come sola o rellena con diversos ingredientes; constituye un elemento esencial en la alimentación mexicana.

4 Es un arbusto que mide de dos a tres metros de alto, posee médula gruesa y blanca, y tallos grisáceos o verdosos. Son ricas en sales de hierro, calcio, vitamina "A" y, sobre todo, vitamina " $\mathrm{C}$ ".

5 Es una proporción de terreno de aproximadamente cuatro mecates (medida aproximada de veinte metros lineales o veinte metros cuadrados), que no se encuentra muy retirado del domicilio familiar. Donde se existen árboles frutales y hortalizas para el consumo familiar, así como cría de animales de traspatio.

6 Las burritas en la comunidad consisten en una preparación con tortilla de harina, untada con mayonesa y jamón (puede ser de cerdo o de pavo) aunque regularmente es de cerdo y de bajo costo, algunas veces puede ir acompañado de queso de hebra.

7 Forma parte de la gastronomía mexicana y se refiere a un tipo de sándwich que se elabora con un pan, como telera o bolillo, el cual se parte por la mitad y se rellena de diferentes alimentos. [] Las tortas pueden servirse frías o calientes. 
por Pérez y colaboradores (21).

En cuanto a la percepción de la imagen corporal son interesantes los datos encontrados ya que existen mujeres y hombres que perciben su imagen dentro de los patrones de normalidad, tal es el caso de las personas de Mucuyché ya que todos(as) aquellos(as) que se perciben obesas la imagen que seleccionaron fue la correspondiente al sobrepeso y no a la obesidad, es decir, anhelan una figura más delgada a la que realmente corresponde, esto puede deberse a que una presión social y al discurso médico occidentalizado sobre la figura "normal", tal como lo menciona Pérez-Gil y Romero, el cuerpo está mediatizado por la cultura y expresa la presión social de cada grupo (22).

De las personas entrevistadas de esta comunidad nadie eligió la figura correspondiente a la obesidad, pero se perciben obesas; esto se puede deber a las actitudes prejuiciosas que se tienen hacia los individuos con sobrepeso y obesos (23). Lo mismo fue reportado por Madrigal y colaboradores en 15 países de la Comunidad Europea (24).

Esta situación fue contraria a la encontrada en Xanláh, en donde son más las personas que se perciben dentro de los patrones normales que con obesidad, esto lo corrobora el IMC detectado, pues el porcentaje de sobrepeso y obesidad en esta localidad es menor en comparación con la otra, es importante mencionar que en esta comunidad nadie se percibe con sobrepeso.

En cuanto a los resultados obtenidos por las entrevistas, las mujeres y hombres mencionan que la obesidad es 'mala' por las enfermedades que se pueden padecer, así como el tener una 'imagen obesa' no es buena, es decir, no cumplen con el patrón de normalidad impuesto por las instituciones sociales e individuales (25), constituyendo un estigma social, así como también cierta preocupación por el peso, resultado de las acciones y del discurso que lleva a cabo el personal médico y de la excesiva propaganda que existe relacionada con lo que se denomina "mercado del adelgazamiento" (22).

Se aprecia en las narraciones que algunas personas que se perciben con sobrepeso y obesidad presentan conformidad con su cuerpo ya que esta condición forma parte de su cultura, de su identidad y belleza, esta situación es más común en las personas adultas y adultas mayores; representando también estatus dentro de su entorno social, ya que las personas que no tienen dinero sólo comen "frijol y maíz", caso contrario con los individuos que tienen acceso económico para comprar "otros alimentos" que ellos(as) considera "buenos", observándose en ambas comunidades que para la personas seleccionan los alimentos no por su valor nutritivo, sino por el estatus, reconocimiento y prestigio que tienen dentro de la comunidad (26), por ello en la dieta habitual se refleja el consumo de alimentos procesados modernos que pueden ser más caros que los alimentos no procesados. Esto es similar a lo reportado por Makkar y Strube (14) así como Bissell (15), quienes en Estados Unidos, encontraron que las mujeres afroamericanas están menos preocupadas y no exigen un patrón ideal del cuerpo, son más tolerantes con la percepción de una imagen corporal con sobrepeso, en comparación con las mujeres caucásicas. Lo que sugiere que se trata principalmente de diferencias raciales y culturales, tal como lo confirman Landrine y Klonoff (16) mencionando que los individuos que conviven con una cultura dominante, puede influir en su propia cultura modificando sus valores, creencia y costumbres (17-20).

\section{CONCLUSIONES}

Se puede evidenciar que las personas eligen una imagen corporal subestimada al peso verdadero que ellos(as) poseen, esto puede ser debido a la presión social y al querer adoptar un concepto totalmente occidental, debido al bombardeo biomédico. También se observa que las familias que están más en contacto con el medio urbano, en este caso, la comunidad el Mucuyché tiende a adoptar un patrón alimentario más industrializado y menos nutritivo, lo que corrobora el porcentaje mayor de sobrepeso y obesidad en comparación con la otra localidad (Xanláh) en donde el patrón alimentario es aún más tradicional, pero en transición.

Es necesario que las políticas públicas en salud consideren las razones del porqué la población está cambiando su alimentación, así como los significados que estas le dan, para que los programas de salud sean orientados a estrategias realmente pertinentes a cada uno de los contextos sociales y culturales del país. También es de suma importancia revalorar el consumo de alimentos que forman parte del cultivo de la milpa, ya que son más sano, nutritivos y de fácil alcance para la población, educando también al buen uso del dinero para la compra de alimentos que posean un valor nutritivo.

\section{RESUMEN}

El objetivo del estudio fue conocer la percepción de la imagen corporal y las prácticas alimentarias de indígenas mayas de dos zonas rurales de Yucatán, México. Se seleccionaron 10 familias por conveniencia hasta que se obtuvo el punto de saturación teórica, con un total de 8 mujeres y 2 hombres de la comunidad de Xanláh (zona maicera), y 8 mujeres y 3 hombres de la localidad de Mucuyché (zona henequenera); se les pesó y midió para identificar el Índice de Masa Corporal (IMC), dieta habitual y entrevistas en profundidad (cuya guía fue validada en un estudio previo) para conocer la percepción de la imagen. Las respuestas mostraron similitudes en ambas comunidades, el estar 'gordito' o 'gordita' forma parte de su contexto cultural y de la "normalidad" $y$, seleccionan los alimentos no por su valor nutritivo, sino por el estatus, reconocimiento y prestigio que dichos alimentos representan. Es indispensable que las políticas se orienten con estrategias pertinentes a los contextos sociales y culturales del país.

Palabras clave: Percepción; tradicional; alimentación; obesidad; sano.

Agradecimientos: El trabajo es el resultado de una tesis doctoral realizada en el programa de Ecología y Desarrollo Sustentable de El Colegio de la Frontera Sur. La autora principal agradece al Consejo Nacional de Ciencia y Tecnología por la beca otorgada para la realización de sus estudios doctorales.

8 Los panuchos son un platillo de la cocina de la Península de Yucatán. Son pequeños tortillas de maíz fritas hechas a mano, a las que se les hace un corte por el que se mete un guiso hecha a base de frijol y que luego son fritas en aceite o manteca de cerdo. Posteriormente se adorna con hojas de lechuga, carne de pavo guisado, tomate y cebolla previamente sazonados con naranja agria y sal, aguacate, zanahoria y recado colorado (achiote). Se pueden acompañar con una cantidad extra de salsa picante hecha a base de chile habanero.

9 Son alimentos industrializados conocidos como "alimentos chatarra" ricos en azúcares simples y grasas saturadas de gran contenido energético.

10 Es una fritura considerada como "alimento chatarra" rica en hidratos de carbono simple, grasa saturada y sal, de gran contenido energético.

11 Es una fritura considerada "alimento chatarra" rica en hidratos de carbono simple, grasa saturada y sal, de gran contenido energético.

12 Es una fritura regional considerada "alimento chatarra" rica en hidratos de carbono simple, grasa saturada y sal, de gran contenido energético. 


\section{BIBLIOGRAFÍA}

1. Popkin M. Doak M. The obesity epidemic is a worldwide phenomenon. Nutr Rev. 1998; 56:106-14.

2. González-Villapalpando C. Stern M. La obe idad como factor de riesgo cardiovascular en México. Estudio en población abierta. Rev Invest Clin. 1993; 45: 13-21.

3. Grundy S. Multifactorial causation of obesity: implications for prevention. Am J Clin Nutr. 1998; 67 (suppl 3): S563-S72.

4. Organización Mundial de la Salud. Informe Mundial. OMS, 2002.

5. UNICEF. Salud y Nutrición. El doble reto de la malnutrición y la obesidad. En http://www.unicef.org/mexico/ spanish/17047.htm

6. Salazar J, Montero M, Muñoz C, Sánchez E, Santero $E$, Villegas J. Percepción social. Ed. Trillas. Psicología social. Asociación Venezolana de Psicología. México, p. 77-108, 2003.

7. Mauss, M. Sociología y Antropología. Tecnos, Madrid, 1979.

8. Williamson $D$, Womble $L$, Zucker $N$, Reas $D$, White $M$, Blouin D, Greenway F. Body image assessment for obesity (BIA-O): development of a new procedure, Int J Obes Relat Metab Disord. 2000; 24: 1326-32.

9. Pérez-Gil S. Romero G. Imagen corporal de mujeres rurales de la Sierra Juárez y Costa de Oaxaca: una aproximación nutrio-antropo-lógica, Estud Soc. 2008; 32: 11-12.

10. Lovejoy M. Disturbances in the Social Body: Differences in Body Image and Eating Problems among African American and White Women, Gender and Society 2001; 15: 239-61.

11. Atalah E, Urteaga C. Rebolledo A. Autopercepción del estado nutricional en adultos de Santiago, Rev Méd Chil. 2004; 132: 1383-8.

12. Instituto Nacional de Estadística y Geografía. Conteo de Población y Vivienda. Volumen II. México. INEGI. 2005.

13. Secretaría de Desarrollo Social. Índice de Marginalidad por Municipios del Estado de Yucatán. México, SEDESOL, 2005.

14. Murguía R. García Q. Los espacios sociales en Yucatán. En Hernández $X$ y Padilla (eds) Seminario de producción agrícola en Mérida, Yucatán: SSP, CP, SARH, Gobierno del estado de Yucatán, p. 83-138, 1982.

15. Lazos E. Del maíz a la naranja en el sur de Yucatán: Auge y dinámica de la huerta. En: Hernández E, Bello E y Levy $S$ (Comp). La milpa en Yucatán. Un sistema de producción agrícola tradicional. Editorial Colegio de Postgraduados. México. p. 527-56.1995.

16. WHO. World Health Organization. Physical status: the use and interpretation of anthropometry. Report of a WHO Expert Committee. WHO Technical Report Series 854. Geneva: World Health Organization, 1995.

17. National Institute of Health. Clinical Guidelines on the Identification, Evaluation, and Treatment of Overweight and Obesity in Adults. Evidence Report. Washington DC, US Department of Health and Human Services, WHO, 1998.

18. Shetty P, James W. Body Mass Index. A Measure of Chronic Energy Deficiency in Adults. Rome, FA, 1994.

19. Pérez-Gil S. Díez S. Estudios sobre alimentación y nutrición en México: una mirada a través del género, Rev. Salud Pública Mex. 2007 49: 445-53.

20. Olaiz G. Rivera J. Shamah T. Rojas R. Villalpando S. Hernández S. Hernández M. Sepúlveda J. Encuesta Nacional de Salud y Nutrición 2006. México, Instituto Nacional de Salud Pública, 2006.

21. Pérez $O$, Nazar $A$, Salvatierra $B$, Pérez-Gil S, Rodríguez $L$, Castillo M, Mariaca R. Frecuencia de consumo de alimentos industrializados modernos en la dieta habitual de comunidades mayas de Yucatán, México. Estudios Sociales. 2012; 39: 155- 84.

22. Pérez-Gil S, Romero G. Imagen corporal en mujeres de tres zonas rurales de México: percepción y deseo, Salud Pública Méx. 2010; 52:111-8.

23. Puhl R. Kelly D. Bias, Discrimination, and Obesity, Obesity Research 2001; 9:788-805.

24. Madrigal $H$, Sánchez-Villegas $A$, Martínez-Gonzáles $M$, Kearney J, Gibney M, Irala J, Et al. Underestimation of body mass index through perceived body image as compared to self-reported body mass index in the European Union. Public Health 2000; 114: 468-73.

25. Carr D, Friedman M. Is Obesity Stigmatizing? Body Weight, Perceived Discrimination, and Psychological Well-Being in the United States. J Health Social Behav 2005; 46: 244-59.

26. Calvo T. López G. Movimientos de población en el Occidente de México. México. Editorial CEMCA, El Colegio de Michoacán. 1993. 